\title{
Are the furanic aldehydes ratio and phenolic aldehydes ratios reliable to assess the addition of vanillin and caramel to the aged wine spirit?
}

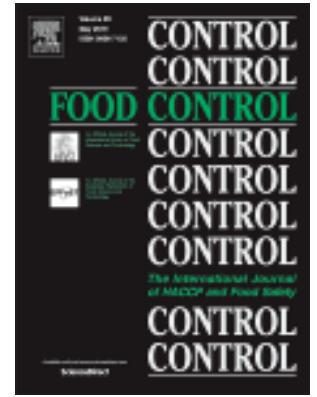

Canas S., Anjos O., Caldeira I., Belchior A. P. 2019. Food Control. 95: 77-84

https://www.sciencedirect.com/science/article/pii/S0956713518303852

\section{Abstract}

European regulations do not allow the addition of flavouring agents, including vanillin, and permit the caramel to be added quantum satis (as a means to adapt colour) to the wine spirit. Therefore, monitoring the authenticity and the caramel content of this beverage in order to prevent unfair competition between producers and to protect the consumer is imperative. This work presents the use of multivariate analysis and control charts, based on the ratios between furanic aldehydes and between phenolic aldehydes, as a simple, rapid, and reliable tool to discriminate aged wine spirits to which vanillin and/or high amount of caramel was added. A representative set of samples, consisting of 288 authentic aged wine spirits collected from trials and 11 commercial brandies, was used to classify 34 commercial aged wine spirits. Despite the high variability inherent to these authentic aged drinks induced by the kind of wood and toasting level, the Factor Analysis performed allowed to firstly separated the authentic samples from the commercial ones, and discriminate the unknown samples of commercial aged wine spirits as authentic or suspect to have been added of vanillin and/or high amount of caramel. The upper control limit (UCL) of the individuals control charts obtained from trials' data - 0.621 for HMF/furfural ratio; 1.165 for vanillin/syringaldehyde ratio; 1.379 for vanillin/coniferaldehyde ratio; 1.438 for vanillin/sinapaldehyde ratio; 0.416 for vanillin/syringaldehyde + coniferaldehyde + sinapaldehyde ratio - proved to be of paramount importance in refining the analysis and identifying the samples with added vanillin (non-authentic) and/or containing high amount of caramel.

\section{Keywords}

Wine spirit; Ageing; Authenticity; Furanic aldehydes ratio; Phenolic aldehydes ratios; Control charts 